\title{
ARTICLE OPEN Rabi oscillations in a superconducting nanowire circuit
}

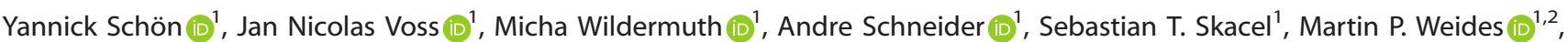 \\ Jared H. Cole $\mathbb{D}^{3}$, Hannes Rotzinger ${ }^{1,4 凶}$ and Alexey V. Ustinov $\mathbb{I D}^{1,5,6}$ \\ We investigate the circuit quantum electrodynamics of anharmonic superconducting nanowire oscillators. The sample circuit \\ consists of a capacitively shunted nanowire with a width of about $20 \mathrm{~nm}$ and a varying length up to $350 \mathrm{~nm}$, capacitively coupled to \\ an on-chip resonator. By applying microwave pulses we observe Rabi oscillations, measure coherence times and the anharmonicity \\ of the circuit. Despite the very compact design, simple top-down fabrication and high degree of disorder in the oxidized (granular) \\ aluminum material used, we observe lifetimes in the microsecond range.
}

npj Quantum Materials (2020)5:18; https://doi.org/10.1038/s41535-020-0220-x

\section{INTRODUCTION}

Quantum electrodynamics experiments with superconducting circuits (CQED) usually feature one or more Josephson tunnel junctions embedded in a circuit. Such circuits often feature a nonlinear inductive response of the Josephson junction, leading to discrete, non-equidistant, energy levels ${ }^{1,2}$. Alternative approaches to the realization of nonlinear elements are, for example, explored using hybrid quantum systems $\mathrm{s}^{3,4}$.

In this paper, we report on a quantum circuit which employs a superconducting nanowire as a nonlinear element. Using the powerful CQED approach, material properties arising at the nanometer scale are studied by directly observing measures like inductance, nonlinearity, or coherence. We demonstrate that such a simple circuit has a rather long $(\sim \mu s)$ excited state lifetime.

As a material we use oxidized (granular) aluminum $\left(\mathrm{AlO}_{x}\right)^{5}$ which has recently been introduced into large impedance quantum circuit applications ${ }^{6}$, due to its low loss properties at microwave frequencies, also in the single photon regime ${ }^{7}$. $\mathrm{AlO}_{x}$ films consist of nanometer-sized aluminum grains (on average of about $4 \mathrm{~nm}$ in diameter) embedded in an insulating aluminum oxide matrix (see e.g. Supplementary Material in ref. ${ }^{5}$ ). The intergrain tunnel barriers lead to a sheet resistance of up to few $k \Omega$ which can be controlled during the film growth by adjusting the oxygen partial pressure.

In general, a wire made from $\mathrm{AlO}_{x}$ can be seen as a series of conducting grains separated by insulating barriers. If the wire width is comparable with the aluminum grain size, its superconducting properties resemble the behavior of a disordered chain of Josephson weak links ${ }^{8}$. Throughout this paper, the studied wires have a width $w$ of about $20 \mathrm{~nm}$.

The nanoscale structure gives rise to the main difference between the nanowire and the Josephson junction. While the lumped Josephson tunnel barrier of a traditional quantum circuit has a sinusoidal current-phase relation, the phase drop along an $\mathrm{AlO}_{x}$ nanowire is distributed over many nanometers and has a more linear current-phase relation involving hundreds of microscopic Josephson weak links. Making use of this nonlinearity for a new type of quantum device poses an intriguing challenge.

In terms of electric loss, nanowires can be advantageous since an applied voltage drops over many junctions and thus the local electric fields are substantially reduced compared to those in a single junction. Therefore, two-level defects present in the vicinity of the local barriers should couple only weakly to the electrical field of such a circuit ${ }^{9,10}$. However, the influence of unpaired spins in the nanoscale granular material ${ }^{11,12}$, e.g. due to the parity effect in the grains, is still to be understood.

From the perspective of a nanowire, two regimes have to be considered. A long and very thin wire with a high normal state resistance $(\gg 50 \mathrm{k} \Omega$ ) undergoes a transition to an insulating state at low temperatures $(T<1 \mathrm{~K})$. Here, due to quantum fluctuations, the superconducting phase is not well defined and, consequently, the electric transport for excitations below the superconducting gap is suppressed. This corresponds to the quantum phase slip regime which has been investigated in the context of homogeneous wires ${ }^{13-16}$. The nanowires considered in this paper instead have a lower normal state resistance and, due to the short coherence length $\left(\xi=(8 \pm 0.4) \mathrm{nm} \simeq w^{17}\right)$, the superconducting phase is well defined in the wire. This means that Cooper pairs can tunnel coherently along the wire up to its critical current $I_{\text {c. }}$. Ignoring the local structure of the wire, this behavior is rather well described by a mean field approach of the Kulik-Omelyanchuk (KO) model ${ }^{17,18}$, which relates $I_{c}$ to the wire's superconducting gap and normal state resistance.

The measurement of quantum coherence in such onedimensional systems constitutes an attractive goal. Transferring this to the high resistance regime could allow for a distinction of dissipative and dissipationless phase slips. Additionally this would give access to intrinsic dynamics and their contributions to the anharmonicity in disordered one-dimensional systems.

\section{RESULTS AND DISCUSSION}

Theory and design

An excitation of the capacitively shunted nanowire having the energy $E=\hbar \omega_{01}$ leads to a current $I \simeq \sqrt{2 E / L}$, where $L$ is the total inductance of the circuit. The nonlinearity of the nanowire depends on current $I$ to the lowest order as $\left(I / I_{c}\right)^{219}$. Therefore, in order to operate the nanowire in a sufficiently anharmonic qubit-like regime, it is useful to keep $/$ as close to $I_{c}$ as possible. This can be achieved by two basic approaches: Either designing

\footnotetext{
${ }^{1}$ Physikalisches Institut, Karlsruhe Institute of Technology, 76131 Karlsruhe, Germany. ${ }^{2}$ School of Engineering, University of Glasgow, Glasgow G12 8QQ, UK. ${ }^{3}$ Chemical and Quantum Physics, School of Science, RMIT University, Melbourne, VIC 3001, Australia. ${ }^{4}$ Institute for Quantum Materials and Technologies, Karlsruhe Institute of Technology, 76131 Karlsruhe, Germany. ${ }^{5}$ National University of Science and Technology MISIS, Moscow 119049, Russia. ${ }^{6}$ Russian Quantum Center, Skolkovo, Moscow 143025, Russia. 莐mail: rotzinger@kit.edu
} 

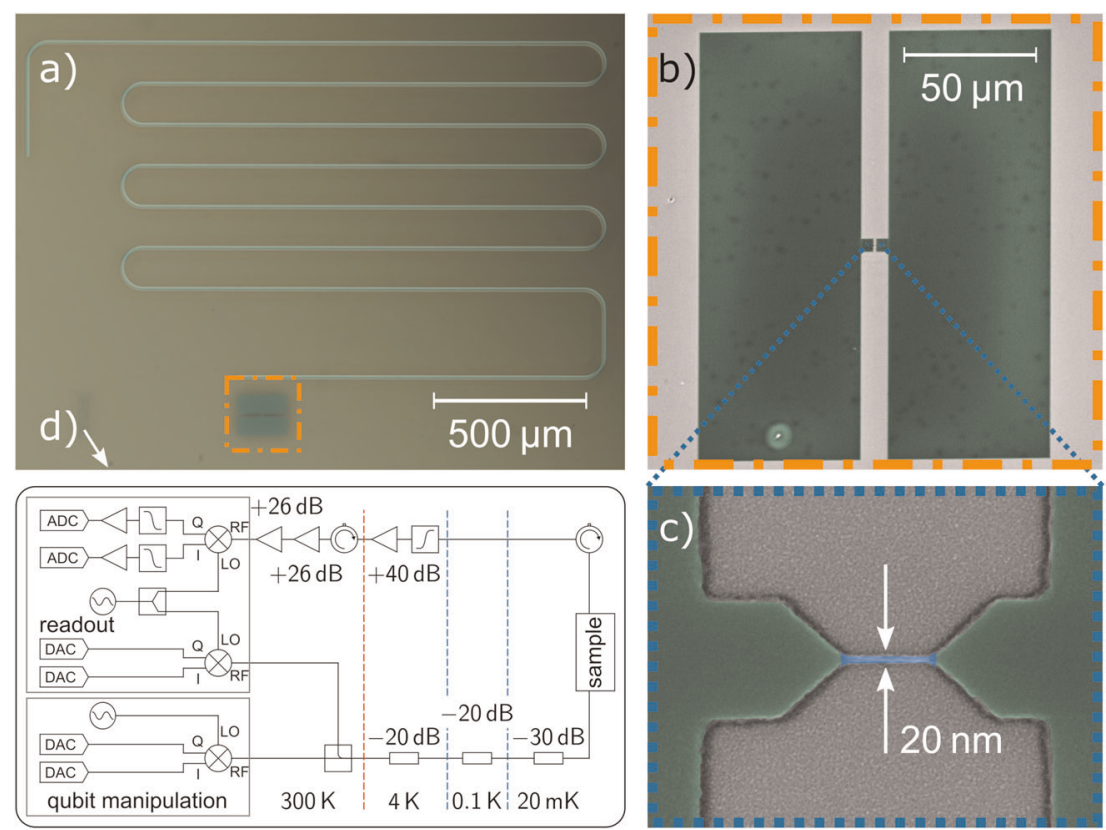

Fig. 1 Sample and setup. a Optical microscopy photograph of a granular aluminum oxide nanowire oscillator (orange border) capacitively coupled to an aluminum resonator (circuit diagram in Supplementary Fig. 1). b Scanning electron microscope (SEM) picture of one nanowire oscillator (colored in green). The large $60 \times 160 \mu \mathrm{m}^{2}$ pads constitute the capacitance. Incremental constrictions lead to the nanowire. c Colored SEM closeup of the nanowire (blue) shunting the two capacitive pads. d Cryogenic microwave measurement setup.

the circuit for a maximal I (low total $L$ ) or operating it with a comparably large inductance $L$ at a much smaller $I_{c}$ (or a combination of both). The first option aims at minimizing the amount of high kinetic inductance material, for instance, by using a conventional low kinetic inductance superconductor like aluminum for the capacitive parts. This approach has the advantage that the geometric requirements for the nanowire are less stringent ${ }^{20,21}$. This, however, leads to a relatively large electric field across the nanowire and thus to potentially increased dielectric losses. We have chosen the second option and fabricated the whole circuit containing a low $I_{c}$ nanowire from a material with a rather large kinetic inductance, thus sacrificing the strength of nonlinearity. The circuit design approach is based on the well known capacitively shunted Josephson junction (transmon-like) qubit ${ }^{22}$, here with substantial differences due to the added large additional kinetic inductance (in the order of a few $\mathrm{nH})$. The large impedance $Z=\sqrt{L / C} \simeq 1 \mathrm{k} \Omega$ of the circuit and the vacuum impedance are thus mismatched to reduce the effects of environmental noise.

The circuit is described by a Hamiltonian similar to the capacitively shunted Josephson junction qubit ${ }^{22,23}$, namely $\mathcal{H}_{\mathrm{T}}=$ $4 E_{\mathrm{C}}\left(\hat{n}-n_{\mathrm{g}}\right)^{2}-E_{\mathrm{J}} \cos \hat{\varphi}$ with charging energy $E_{\mathrm{C}}=e^{2} / 2 C$ and Josephson coupling energy $E_{J}=\Phi_{0} l_{c} / 2 \pi$. In a nanowire, the current-phase relation is non-sinusoidal and has a form, which we denote as $I \sim f^{\prime}(\varphi)$. While its exact form is unknown, from the studies of superconducting weak links we anticipate it to be a function shaped between sine and sawtooth form (see e.g. refs ${ }^{24-26}$ ). We assume orders of magnitude larger critical currents in the capacitive part of the circuit and thus neglect the nonlinearity of the kinetic inductance contribution there. We split the Josephson coupling term in the Hamiltonian into a linear part and the contribution of an effective junction (circuit design and diagram in Supplementary Note and Fig. 1). Following the circuit quantization $^{27}$ approach, a Hamiltonian can be written as $\mathcal{H}=4 E_{\mathrm{C}}\left(\hat{n}-n_{\mathrm{g}}\right)^{2}-\tilde{E}_{\mathrm{J}} f(\hat{\varphi})+\tilde{E}_{\mathrm{L}} \hat{\varphi}^{2}$. Here $\tilde{E}_{\mathrm{J}}=6 E_{\mathrm{J}} E_{\mathrm{L}}^{2} /\left(6 E_{\mathrm{J}}+2 E_{\mathrm{L}}\right)^{2}$ and $\tilde{E}_{\mathrm{L}}=9 E_{J}^{2} E_{\mathrm{L}} /\left(6 E_{\mathrm{J}}+2 E_{\mathrm{L}}\right)^{2} . \quad E_{\mathrm{L}}$ is the inductive energy $\left(\Phi_{0} / 2 \pi\right)^{2} / 2 L$. This is valid in the limit of $E_{\mathrm{L}}$ being small compared to all other energy terms ${ }^{28}$. To achieve a sufficient nonlinearity of the circuit, the wire has to be long enough as to ensure it dominates the inductance of the system. It remains, however, to be studied how the wire length influences the nonlinearity of the current-phase relation.

The nanowire is capacitively shunted by two rectangular $60 \times$ $160 \mu \mathrm{m}$ capacitor pads, with a distance of $10 \mu \mathrm{m}$ (see Fig. 1). As obtained by analytical calculation ${ }^{29}$ and simulation (ANSYS Maxwell), the pads constitute a $(22 \pm 2) \mathrm{fF}$ capacitance. The studied three nanowire circuits have varied wire lengths of 50, 100 , and $350 \mathrm{~nm}$ and are coupled to individual readout resonators at $6.85,6.10$, and $4.99 \mathrm{GHz}$. For a summary of the sample parameters see Table 1.

\section{Measurements}

Three anharmonic oscillators with varying wire length were measured using a dispersive readout scheme ${ }^{30}$. Transition frequencies $f_{01}$ were determined spectroscopically by observing the shift of the readout resonator's frequency induced by the second drive tone. The circuit's inductance can be derived from its normal conductive resistance as $L=0.18 \hbar R /\left(k_{\mathrm{B}} T_{\mathrm{c}}\right)$, where $T_{\mathrm{c}}$ is about $1.8 \mathrm{~K}^{5}$. With the capacitance $C$, a harmonic approximation for the circuit's frequency is $f_{\text {calc. }}=1 /(2 \pi \sqrt{L C})$. While this estimate agrees with the measured transition frequency of sample S1 (350 $\mathrm{nm}$ long wire), for samples S2 $(50 \mathrm{~nm})$ and S3 $(100 \mathrm{~nm})$ the measured transition frequency is lower than the estimate. The later two samples also exhibit higher room temperature wire resistances and a higher wire to pad resistance ratio (Table 1 ).

A continuous microwave tone on resonance with a system's transition frequency results in Rabi oscillations between its ground and excited states. These oscillations are recorded using pulsed time-domain measurements. Figure $2 \mathrm{a}$ depicts the time evolution of the circuit excitation in dependence of the drive amplitude, measured in sample S2. Brighter colors correspond to the system being excited. The microwave drive amplitude has been calibrated after the room temperature part of the microwave setup (Fig. 1) with a spectrum analyzer. This compensates for the nonlinearities of the IQ mixers. In case of a single two-level transition coupled to 
Table 1. Properties of the sample circuits.

\begin{tabular}{lllllll}
\hline Sample & $I_{\mathrm{W}}(\mathrm{nm})$ & $R_{\mathrm{W}}(\mathrm{k} \Omega)$ & $f_{\mathrm{r}}(\mathrm{GHz})$ & $f_{01}(\mathrm{GHz})$ & $f_{\text {calc. }}(\mathrm{GHz})$ & $5.2 \pm 0.3$ \\
\hline S1 & 350 & $<7$ & 4.99 & 5.47 & $9.2 \pm 0.4$ & $3.4 \pm 0.1\left(0.8 \pm 0.2 T_{2}\right)$ \\
S2 & 50 & $7 \pm 1$ & 6.85 & 8.50 & $8.0 \pm 0.1$ \\
S3 & 100 & $10 \pm 1$ & 6.10 & 7.93 & $8.3 \pm 0.4$ & $3.4 \pm 0.1$ \\
\hline
\end{tabular}

The wire length is denoted by $I_{\mathrm{W}} . R_{\mathrm{W}}$ is the room temperature resistance of the wire estimated from the total circuit resistance. The readout resonator frequencies $f_{\mathrm{r}}$ and transition frequencies $f_{01}$ are obtained from spectroscopy. $f_{\text {calc. }}$ corresponds to the harmonic estimate of the circuits resonance. The lifetimes are obtained by time-domain measurements.
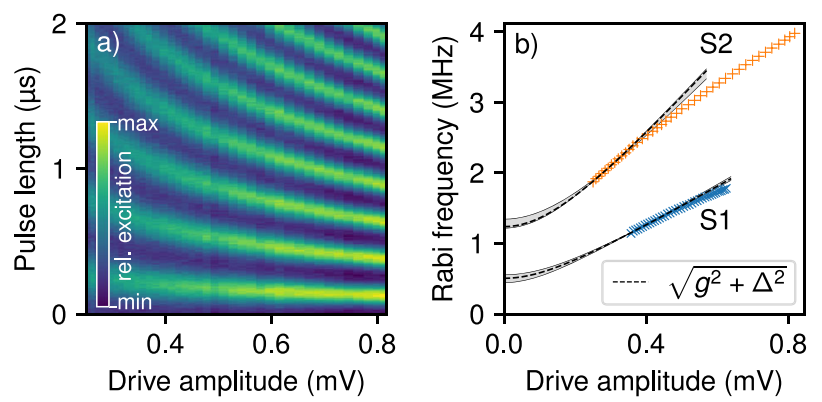

Fig. 2 Rabi oscillation power dependence. a Rabi oscillations measured in sample S2 with varied drive amplitude. b The fitted Rabi frequencies measured in S1 as well as S2 fit to the two-level Rabi behavior until they deviate at frequencies exceeding the anharmonicity. The values for the drive detuning $\Delta$ obtained from the fit are $\Delta_{\mathrm{S} 1}^{\mathrm{fit}}=(0.5 \pm 0.1) \mathrm{MHz}$ and $\Delta_{\mathrm{S} 2}^{\mathrm{fit}}=(1.3 \pm 0.1) \mathrm{MHz}$. Errorbands include a variation of the number of points fitted between 6 and 16. Compared with spectroscopy the Rabis were driven with $\Delta_{\mathrm{S} 1}=(0.8 \pm 0.5) \mathrm{MHz}$ and $\Delta_{\mathrm{S} 2}=(1.8 \pm 1) \mathrm{MHz}$, respectively. In sample S1, a smaller anharmonicity agrees with a lower normal state wire resistance (Table 1).

a radiative field, the oscillation frequency $\Omega$ is expected to depend linearly on the coupling $g$, which is proportional to the driving field amplitude. An additional small detuning $\Delta$ between the drive and transition frequencies results in the form $\Omega=\sqrt{g^{2}+\Delta^{2}} 31$. The Rabi frequency $\Omega$ is extracted from damped sine fits and displayed in Fig. 2b. In both depicted oscillators S1 (350 nm long wire) and S2 (50 nm long wire), $\Omega$ fits to the two-level Rabi behavior until it starts to deviate toward higher frequencies. This deviation is expected in the presence of higher levels when $\Omega$ exceeds the circuit's anharmonicity, given by the difference between the lowest energy level separations ${ }^{32,33}$. In this regime, multi-photon transitions to higher levels occur with a higher rate, thus reducing the power proportion driving the main transition.

Direct spectroscopy of multi-photon transitions to higher levels requires increasing drive powers ${ }^{34}$. In the case of a small anharmonicity, the resulting broadening of the fundamental resonance line makes multi-photon resonances difficult to resolve (Supplementary Note 2). However, by observing Rabi oscillations with varied drive frequency, effects of higher transitions manifest themselves in a distinct asymmetry (Fig. 3a). At frequencies above the main transition, the Rabi oscillation frequency $\Omega$ increases as its amplitude decreases as expected from off-resonant driving. Toward lower frequencies however, $\Omega$ continues to decrease until the oscillation breaks down. Also the oscillation amplitude increases and the equilibrium excitation for long driving rises. These effects agree with a system exhibiting a small but non-vanishing, negative anharmonicity between 1 and $2 \mathrm{MHz}$, allowing the drive to excite the fundamental transition and transitions to higher levels.

The presented interpretation of the above described effects is supported by numerical simulation of the Lindblad-GKS master equation ${ }^{35,36}$, see Supplementary Note 3 . The system $\mathcal{H}_{0}=$
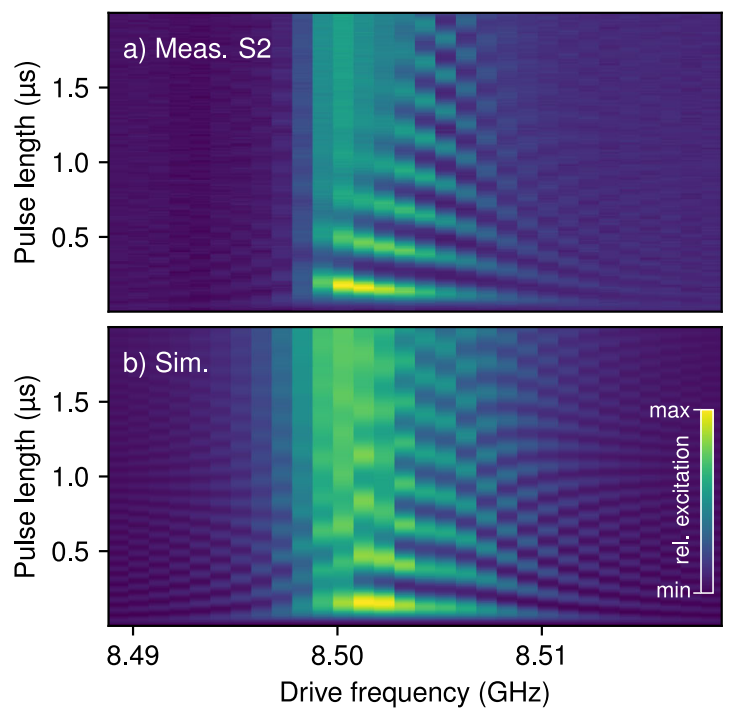

Fig. 3 Rabi oscillation frequency dependence. a Rabi oscillations measured in S2 ( $50 \mathrm{~nm}$ long wire) in a span of $30 \mathrm{MHz}$ around the low-power transition frequency of $8.504 \mathrm{GHz}$. The distinct asymmetry can be attributed to higher level excitations and a relative anharmonicity between 1 and $2 \mathrm{MHz}$. b Result of the numerical Floquet matrix calculation of the density matrix master equation for an anharmonic oscillator with $1.3 \mathrm{MHz}$ anharmonicity. This agrees with an estimation from Fig. 2.

$h\left(f_{01}+f_{\mathrm{s}} \sqrt{\epsilon^{2} /\left(\epsilon^{2}+\Delta^{2}\right)}\right) \hat{n}-h f_{\text {an }}\left(\hat{n}^{2}-\hat{n}\right)$ with the bosonic number operator $\hat{n}$ describes an oscillator with frequency $f_{01}$ and anharmonicity $f_{a n}$. An additional asymmetry extending toward the off-resonant regions in the measurement is accounted for by a slight shift $f_{\mathrm{s}}$ of the main transition in a region defined by a parameter for the width $\epsilon$ and reduced with the detuning $\Delta$ of the drive. This shift is expected due to an AC Stark effect ${ }^{37}$. In this Lindbladian approach ${ }^{35,38}$, two decay channels with corresponding rates are assumed to account for energy dissipation and dephasing. Here we are specifically interested in the interplay between finite anharmonicity and multi-photon (strong driving) effects. Therefore a Floquet expansion ${ }^{39,40}$ is used to solve for the time evolution in the strongly periodically driven system (see Supplementary Note 3).

To reproduce the measurement performed on sample S2 by the simulation shown in Fig. 3b, the main transition frequency and excitation lifetime were taken from separate measurements (Supplementary Notes 2 and 3). The region around the main transition is well reproduced assuming the anharmonicity of $(1.5 \pm 0.3) \mathrm{MHz}$.

Excitation decay times $T_{1}$ were measured by applying a microwave pulse $t_{\pi}$ of half a Rabi period. Measurement of the excitation after a varied delay yields an exponential decay $\propto \mathrm{e}^{-t / T_{1}}$. The $T_{1}$ times, measured in all samples, range between 3.4 and $4 \mu$ s (Fig. 4a). 


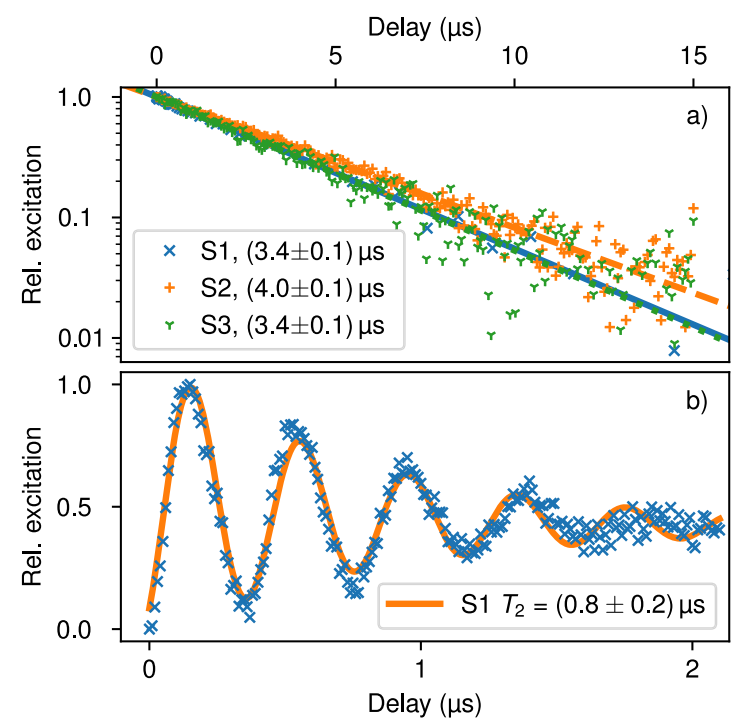

Fig. 4 Lifetime measurements. a Excitation lifetimes $T_{1}$ measured in samples S1, S2, and S3 range between 3.4 and $4 \mu \mathrm{s}$. b Ramsey fringes measured in $\mathrm{S} 1$ yield a dephasing time $T_{2}$ of $(0.8 \pm 0.2) \mu \mathrm{s}$.

Excitation to a state on the equator of the Bloch sphere is possible by applying a $t_{\pi / 2}$-pulse. Off-resonant driving and varying the delay before applying the second $t_{\pi / 2}$-pulse results in Ramsey fringes (Fig. 4b). The decay of the fringes corresponds to the dephasing time $T_{2}$. Our measurement performed in sample S1 yielded a $T_{2}$ of $0.82 \mu \mathrm{s}$.

We estimated single-mode Purcell loss $\gamma_{\mathrm{p}}=\left(g_{\mathrm{r}} / \Delta_{\mathrm{r}}\right)^{2} \kappa$ (refs ${ }^{41,42}$ ) given by the coupling $g_{\mathrm{r}}=(17 \pm 2) \mathrm{MHz}$ to the readout resonator, its linewidth $K=(1.5 \pm 0.2) \mathrm{MHz}$ and the frequency detuning $\Delta_{\mathrm{r}}=$ $(487 \pm 1) \mathrm{MHz}$ for sample S1. The obtained Purcell lifetime limitation is $(85 \pm 14) \mu \mathrm{s} \gg T_{1}$. The large circuit impedance favors suppressing radiative loss. No direct correlation between transition frequency and lifetime has been observed. The contribution of dielectric loss due to randomly distributed two-level systems, which are always present in quantum circuits, can be more easily resolved with higher anharmonicity, as well as temperature- or strain-dependent studies ${ }^{42-44}$.

We expect that transitions to higher levels, which occur at frequencies close to the fundamental transition, contribute additional loss channels. This situation is facilitated by the finite bandwidth of the short control pulses. A rectangular $100 \mathrm{~ns}$ long $\pi$-pulse has a linewidth that can be estimated as $1 /(2 \pi \cdot 100 \mathrm{~ns})=$ $1.6 \mathrm{MHz}$, thus being of the order of the observed anharmonicity. Optimized pulse shapes can help decrease the rate of unintended excitations ${ }^{45}$. Excitations of higher transitions lead to additional frequency components modulating Ramsey fringes and thus make a precise measurement of the pure dephasing challenging. Also, charge noise due to a large charging energy $e^{2} / 2 h C$ of $(880 \pm 80)$ $\mathrm{MHz}$ adds to the dephasing in the circuits. This contribution can be reduced together with a decrease of the total inductance by concentrating the circuit's inductance in the wire. A thus increased anharmonicity would allow for a distinction of the higher level contribution to the observed lifetimes and thus help anticipate the feasible performance of optimized $\mathrm{AlO}_{x}$ nanowire qubits.

\section{CONCLUSION}

In this work, we demonstrated multi-level quantum dynamics in single-layer superconducting circuits. The anharmonic nature of these nanowire oscillators originates from nonlinear properties of the material they are made of, oxidized (granular) aluminum. These nanowire quantum circuits feature a simple, scalable fabrication process and are compact in design. The fabricated samples showed energy relaxation times $T_{1}$ of up to $4 \mu$ s and a relatively small negative anharmonicity on the order of $1-2 \mathrm{MHz}$. The measured characteristic Rabi patterns were replicated in numerical simulations. We thus demonstrated that nonlinearity and coherence times can be useful measures of the properties of granular materials structured into nanometer size circuits.

In the future, larger anharmonicities seem feasible through featuring nanowires with controllable smaller critical currents and reduced spread. To reduce the contribution of the capacitive paddles to the linear fraction of kinetic inductance, one can use additional shunts made of pure aluminum. These improvements would additionally enhance the circuit's usability as qubit. Studying of the system's Hamiltonian in dependence of the nanowire's geometry and resistance would be facilitated by direct spectroscopy of the level structure. This would shed further light on the physics of the nonlinearity arising from the granular material in such systems.

\section{METHODS}

The disordered oxidized aluminum thin films are grown on a c-plane sapphire substrate by sputter deposition. Details of the process can be found in ref. ${ }^{5}$ In addition, an in-situ resistance monitoring was used to allow for a precise control over the final sheet resistance of the film $(\mathrm{k} \Omega$ range, $20 \mathrm{~nm}$ film thickness) ${ }^{46}$.

The nanowire circuits are defined in a single electron beam lithography step using a bilayer resist stack of hydrogen silsesquioxane (HSQ) and polymethylmethacrylat (PMMA) on top of the $\mathrm{AlO}_{x}$ thin film. This approach has the advantage that the high-resolution HSQ resist can be lifted off after the pattern transfer with an organic solvent. The pattern transfer into the $\mathrm{AlO}_{x}$ thin film is carried out using an anisotropic oxygen and argon/chlorine reactive ion plasma. The aluminum resonators, feedline and backside metallization, are deposited using optical lithography and the lift-off technique.

Due to the galvanic isolation of the nanowire circuits, we characterized the DC resistance at room temperature using needle probes. This method poses severe limitations on the DC measurement results since it is sensitive to the contact resistance and position of the needles on the thin film sample. Special care has to be taken to avoid scratches. Additionally, the wire resistance can be altered by too high probe currents during the resistance measurement ${ }^{17}$.

Microwave spectroscopy of the circuits as well as pulsed time-domain measurements are performed in a dilution cryostat at $20 \mathrm{mK}$ (Fig. 1d).

\section{DATA AVAILABILITY}

The data that support the findings of this study are available from the corresponding author upon reasonable request.

\section{CODE AVAILABILITY}

Measurements and data analysis in the context of this paper were performed with the open source software toolkit Qkit (https://git.io/qkit). The code for the numerical modeling is available from the corresponding author upon reasonable request.

Received: 13 September 2019; Accepted: 21 February 2020; Published online: 13 March 2020

\section{REFERENCES}

1. Makhlin, Y., Schön, G. \& Shnirman, A. Quantum-state engineering with Josephson-junction devices. Rev. Mod. Phys. 73, 357-400 (2001).

2. Devoret, M. H., Wallraff, A. \& Martinis, J. M. Superconducting qubits: a short review. Preprint at http://arxiv.org/abs/cond-mat/0411174 (2004).

3. Larsen, T. W. et al. Semiconductor-nanowire-based superconducting qubit. Phys. Rev. Lett. 115, 127001 (2015).

4. Wang, J. I.-J. et al. Coherent control of a hybrid superconducting circuit made with graphene-based van der Waals heterostructures. Nat. Nanotechnol. 14, 120-125 (2019).

5. Rotzinger, $\mathrm{H}$. et al. Aluminium-oxide wires for superconducting high kinetic inductance circuits. Superconductor Sci. Technol. 30, 025002 (2016).

6. Grünhaupt, L. et al. Granular aluminium as a superconducting material for highimpedance quantum circuits. Nat. Mater. 18, 816-819 (2019). 
7. Grünhaupt, L. et al. Loss mechanisms and quasiparticle dynamics in superconducting microwave resonators made of thin-film granular aluminum. Phys. Rev. Lett. 121, 117001 (2018).

8. Maleeva, N. et al. Circuit quantum electrodynamics of granular aluminum resonators. Nat. Commun. 9, 3889 (2018).

9. Grabovskij, G. J., Peichl, T., Lisenfeld, J., Weiss, G. \& Ustinov, A. V. Strain tuning of individual atomic tunneling systems detected by a superconducting qubit. Science 338, 232-234 (2012).

10. Bilmes, A. et al. Electronic decoherence of two-level systems in a Josephson junction. Phys. Rev. B 96, 064504 (2017).

11. Bachar, N., Lerer, S., Hacohen-Gourgy, S., Almog, B. \& Deutscher, G. Kondo-like behavior near the metal-to-insulator transition of nanoscale granular aluminum. Phys. Rev. B 87, 214512 (2013).

12. Barone, C. et al. Kondo-like transport and magnetic field effect of charge carrier fluctuations in granular aluminum oxide thin films. Sci. Rep. 8, 13892 (2018).

13. Lau, C. N., Markovic, N., Bockrath, M., Bezryadin, A. \& Tinkham, M. Quantum phase slips in superconducting nanowires. Phys. Rev. Lett. 87, 217003 (2001).

14. Mooij, J. E. \& Harmans, C. J. P. M. Phase-slip flux qubits. N. J. Phys. 7, 219-219 (2005).

15. Mooij, J. E. \& Nazarov, Y. V. Superconducting nanowires as quantum phase-slip junctions. Nat. Phys. 2, 169-172 (2006).

16. Astafiev, O. V. et al. Coherent quantum phase slip. Nature 484, 355-358 (2012).

17. Voss, J. N. et al. In preparation (2020).

18. Kulik, I. O. \& Omel'Yanchuk, A. N. Contribution to the microscopic theory of the Josephson effect in superconducting bridges. J. Exp. Theor. Phys. Lett. 21, 96 (1975).

19. Anlage, S., Snortland, H. \& Beasley, M. A current controlled variable delay superconducting transmission line. IEEE Trans. Magn. 25, 1388-1391 (1989).

20. Vijay, R., Sau, J. D., Cohen, M. L. \& Siddiqi, I. Optimizing anharmonicity in nanoscale weak link Josephson junction oscillators. Phys. Rev. Lett. 103, 087003 (2009).

21. Levenson-Falk, E. M., Vijay, R. \& Siddiqi, I. Nonlinear microwave response of aluminum weak-link Josephson oscillators. Appl. Phys. Lett. 98, 123115 (2011).

22. Koch, J. et al. Charge-insensitive qubit design derived from the Cooper pair box. Phys. Rev. A 76, 042319 (2007).

23. Schreier, J. A. et al. Suppressing charge noise decoherence in superconducting charge qubits. Phys. Rev. B 77, 180502 (2008).

24. Christiansen, P. V., Hansen, E. B. \& Sjöström, C. J. Negative self-inductance in superconducting thin wires and weak links. J. Low. Temp. Phys. 4, 349-389 (1971)

25. Likharev, K. K. Superconducting weak links. Rev. Mod. Phys. 51, 101-159 (1979).

26. Wilkinson, S. A., Vogt, N., Golubev, D. S. \& Cole, J. H. Approximate solutions to Mathieu's equation. Phys. E: Low.-dimensional Syst. Nanostruct. 100, 24-30 (2018).

27. Devoret, M. H. Quantum fluctuations in electrical circuits. In Quantum Fluctuations (Les Houches, Session LXIII) (eds Reynaud, S., Giacobino, E. \& David, F.) 351-386 (North-Holland, 1997).

28. Braumüller, J. et al. Concentric transmon qubit featuring fast tunability and an anisotropic magnetic dipole moment. Appl. Phys. Lett. 108, 032601 (2016).

29. Gevorgian, S. \& Berg, H. Line capacitance and impedance of coplanar-strip waveguides on substrates with multiple dielectric layers. In 31st European Microwave Conference, 2001 (IEEE, 2001).

30. Blais, A., Huang, R.-S., Wallraff, A., Girvin, S. M. \& Schoelkopf, R. J. Cavity quantum electrodynamics for superconducting electrical circuits: an architecture for quantum computation. Phys. Rev. A 69, 062320 (2004).

31. Nielsen, M. A. \& Chuang, I. L. Quantum computation and quantum information: 10th Anniversary Edition. 10th edn (Cambridge University Press, New York, NY, USA, 2011)

32. Claudon, J., Balestro, F., Hekking, F. W. J. \& Buisson, O. Coherent oscillations in superconducting multilevel quantum system. Phys. Rev. Lett. 93, 187003 (2004).

33. Dutta, S. K. et al. Multilevel effects in the Rabi oscillations of a Josephson phase qubit. Phys. Rev. B 78, 104510 (2008).

34. Braumüller, J. et al. Multiphoton dressing of an anharmonic superconducting many-level quantum circuit. Phys. Rev. B 91, 054523 (2015).

35. Lindblad, G. On the generators of quantum dynamical semigroups. Commun Math. Phys. 48, 119-130 (1976).

36. Gorini, V., Kossakowski, A. \& Sudarshan, E. C. G. Completely positive dynamical semigroups of N-level systems. J. Math. Phys. 17, 821-825 (1976).

37. Schneider, A. et al. Local sensing with the multilevel ac Stark effect. Phys. Rev. A 97, 062334 (2018).

38. Gardiner, C. \& Zoller, P. Quantum Noise: A Handbook of Markovian and NonMarkovian Quantum Stochastic Methods with Applications to Quantum Optics. 3 edn (Springer-Verlag, Berlin, Heidelberg, 2004).

39. Shirley, J. H. Solution of the Schrödinger equation with a Hamiltonian periodic in time. Phys. Rev. 138, B979-B987 (1965).

40. Bain, A. D. \& Dumont, R. S. Introduction to Floquet theory: the calculation of spinning sideband intensities in magic-angle spinning NMR. Concepts Magn. Reson. 13, 159-170 (2001).
41. Purcell, E. M. Spontaneous emission probabilities at radio frequencies. In Proceedings of the American Physical Society, Phys. Rev. 69, 681 (1946).

42. Houck, A. A. et al. Controlling the spontaneous emission of a superconducting transmon qubit. Phys. Rev. Lett. 101, 080502 (2008).

43. Goetz, J. et al. Second-order decoherence mechanisms of a transmon qubit probed with thermal microwave states. Quantum Sci. Technol. 2, 025002 (2017).

44. Müller, C., Cole, J. H. \& Lisenfeld, J. Towards understanding two-level-systems in amorphous solids: insights from quantum circuits. Rep. Prog. Phys. 82, 124501 (2019).

45. Motzoi, F., Gambetta, J. M., Rebentrost, P. \& Wilhelm, F. K. Simple pulses for elimination of leakage in weakly nonlinear qubits. Phys. Rev. Lett. 103, 110501 (2009).

46. Wildermuth, M. et al. In preparation (2020).

\section{ACKNOWLEDGEMENTS}

The authors want to thank J. Lisenfeld and I. Pop for fruitful discussions as well as L. Radtke and S. Diewald for assistance in sample fabrication and R. Gebauer for contributions to the measurement setup. This work was supported by the Initiative and Networking Fund of the Helmholtz Association, as well as by the Helmholtz International Research School for Teratronics (to Y.S. and J.N.V.), the Landesgraduiertenförderung of the federal state Baden-Württemberg (to M.W.) and the Car Zeiss Foundation (to A.S.). This work was partially supported by the Ministry of Education and Science of the Russian Federation in the framework of the Program to Increase Competitiveness of the NUST MISIS (contract No. K2-2017-081). J.H.C. acknowledges support of the Australian Research Council Centre of Excellence funding scheme (CE170100039) and the NCI National Facility through the National Computational Merit Allocation Scheme. M.P.W. acknowledges support of the European Research Council (ERC) under the Grant Agreement 648011. We acknowledge support by the KIT-Publication Fund of the Karlsruhe Institute of Technology.

\section{AUTHOR CONTRIBUTIONS}

All authors contributed to discussions and interpretations of the results. The experiment was conceived by Y.S. and H.R. Measurements were performed by Y.S. with support by A.S. and M.P.W. Fabrication was done and supported by Y.S., J.N.V., M.W., S.T.S., and H.R.; J.H.C. additionally advised the numerical modeling. The project was supervised by H.R. and A.V.U.

\section{COMPETING INTERESTS}

The authors declare no competing interests.

\section{ADDITIONAL INFORMATION}

Supplementary information is available for this paper at https://doi.org/10.1038/ s41535-020-0220-x.

Correspondence and requests for materials should be addressed to H.R.

Reprints and permission information is available at http://www.nature.com/ reprints

Publisher's note Springer Nature remains neutral with regard to jurisdictional claims in published maps and institutional affiliations.

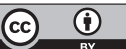

Open Access This article is licensed under a Creative Commons Attribution 4.0 International License, which permits use, sharing, adaptation, distribution and reproduction in any medium or format, as long as you give appropriate credit to the original author(s) and the source, provide a link to the Creative Commons license, and indicate if changes were made. The images or other third party material in this article are included in the article's Creative Commons license, unless indicated otherwise in a credit line to the material. If material is not included in the article's Creative Commons license and your intended use is not permitted by statutory regulation or exceeds the permitted use, you will need to obtain permission directly from the copyright holder. To view a copy of this license, visit http://creativecommons. org/licenses/by/4.0/.

(c) The Author(s) 2020 